\title{
Texture Analysis by the
} Measurement of Individual Grain Orientations-Electron Microscopical Methods and Application on Dual-Phase Steel

\author{
R. A. SCHWARZER and H. WEILAND \\ Institut für Metallkunde und Metallphysik der TU Clausthal, FRG
}

(Received October 30, 1987; in final form December 17, 1987)

\section{Dedicated to the memory of Professor Günter Wassermann}

A short review is given on electron microscopical methods for the determination of local textures. Electron microscopy offers the unique facility of diffraction from well defined locations in the sample and high resolution imaging of the microstructure. Standard techniques of orientation determination are the evaluation of electron diffraction spot and-more recently-Kikuchi patterns, which are applicable to electron transparent fine-grain materials. Specimen areas smaller than $500 \mathrm{~nm}$ or $50 \mathrm{~nm}$ in diameter, respectively, can be selected. Bulk samples with crystallite sizes greater than approximately $1 \mu \mathrm{m}$ or $5 \mu \mathrm{m}$, respectively, can be studied with the SEM using electron backscattering or channelling patterns. For routine work a program has been developed to do on-line determination of orientation of cubic, orthorhombic or hexagonal crystals from Kikuchi, channelling and backscattering patterns.

Applications of this technique on dual-phase steel are given. The orientation distribution functions (ODF) of the ferrite and martensite phases from individual grain measurements are compared with $\mathrm{X}$-ray and neutron pole-figure measurements. Misorientation distribution functions (MODF) are discussed for contiguous ferrite-martensite and martensite-martensite grains.

KEY WORDS: Electron diffraction, selected area electron diffraction (SAD), microbeam diffraction, Kikuchi patterns, channelling patterns, backscattering patterns, ODF-analysis, misorientation distribution function (MODF), dual-phase steels. 


\section{INTRODUCTION}

Preferred crystal orientations ("texture") are usually determined using X-ray or neutron diffraction methods which integrate over a large volume of the sample and hence over a large number of grains, owing to the high depth of penetration. This is an adequate procedure for most applications since texture determination is a question of statistics which is intended to give information on the macroscopic behaviour or the previous history of the bulk material (Bunge, 1982). There are, however, some applications where the knowledge of local textures of areas far below $100 \mu \mathrm{m}$ diameter or even the orientations of individual grains is required, e.g. for a detailed understanding of deformation, recrystallisation and grain growth. With electron diffraction, unlike X-ray or neutron polefigure measurements, it is possible to obtain information on a very localized region simply because the lens systems allow the electron beam to be focussed to a small spot which defines the sampled area on the specimen.

\section{THE EVALUATION OF INDIVIDUAL GRAIN ORIENTATIONS}

\subsection{Selected-area diffraction in transmission electron microscopy (TEM)}

The classical technique for obtaining a diffraction pattern in the TEM is to illuminate the specimen with a parallel beam of electrons, then to select, with a diaphragm in the intermediate image plane, an area of interest in the enlarged image, and finally to display a diffraction pattern from this area by focussing the back focal objective plane onto the fluorescent screen (selected-area electron diffraction, named "SAD"). Lateral resolution in SAD cannot be improved to any extent by simply stopping down the size of the selected area. As a consequence of the spherical and chromatic aberrations of the objective lens, and focussing errors, the individual dark field images which are formed by the diffracted beams are slightly displaced on the selector diaphragm with respect to each other and to the bright-field image. The terms "bright-field" 
and "dark-field" refer, respectively, to images to which the forwardscattered ("primary") beam does or does not contribute. An additional displacement of the selected area in the specimen may result from transverse stray fields when switching the intermediate lens from imaging to diffraction mode (Riecke, 1961). In modern transmission microscopes the smallest selectable area in SAD is in the range of 200 to $500 \mathrm{~nm}$.

If the selected area is a single crystal, the diffraction pattern consists of bright spots ("spot pattern") which are usually interpreted as being the intersections between the Ewald sphere and the reciprocal-lattice points of the crystallite. Since the sample must be relatively thin to be transparent for electrons, the reciprocal lattice points are elongated along the foil-normal to become ellipsoids in reciprocal space. They may be intersected by the Ewald sphere even when the exact Bragg condition is not met and thus contribute to the intensity of the corresponding diffraction spots. The result of this possible deviation is some loss of precision in the determination of grain orientation. In principle, accuracy of orientation may be improved from several degrees in the usual mode of application to about $0.5^{\circ}$ if not only the positions but also the intensities of the diffraction spots are evaluated ("method of intensity centre"; (Laird et al., 1966)). The lack of accuracy results in an incorrect description of crystal orientation. Low-index orientations are emphasized so that real orientation distributions and particularly orientation relationships are obscured. An example is discussed in section 6.

\subsection{Microbeam diffraction in the transmission electron microscope}

In modern transmission electron microscopes, particularly those dedicated to analytical or scanning transmission applications, the spot size of the focussed beam on the specimen can be reduced by more than one order of magnitude beyond the smallest selectable region in SAD and, furthermore, the beam deflection coils can be used for positioning the spot on selected locations on the specimen ("microbeam electron diffraction" (MBD); "Feinstrahlbeugung" (Riecke, 1962)). The angle of convergence of the primary beam is of little consequence here. The often used term "convergent beam 
electron diffraction" (CBED) should be reserved for applications when a large angle of convergence is needed, e.g. KosselMöllenstedt or HOLZ diffraction patterns. With an intense small electron spot a large angle of convergence cannot be avoided. Hereby disk-shaped rather than dotted diffraction spots, as found with parallel illumination in SAD, are formed. This effect should lead to a further loss of accuracy in evaluating crystal orientation. Fortunately the tendency of Kikuchi patterns to form even with thin samples is increased as the beam is made more convergent.

For a detailed understanding of the formation of Kikuchi patterns the dynamical theory of electron diffraction (Whelan, 1970; Reimer,

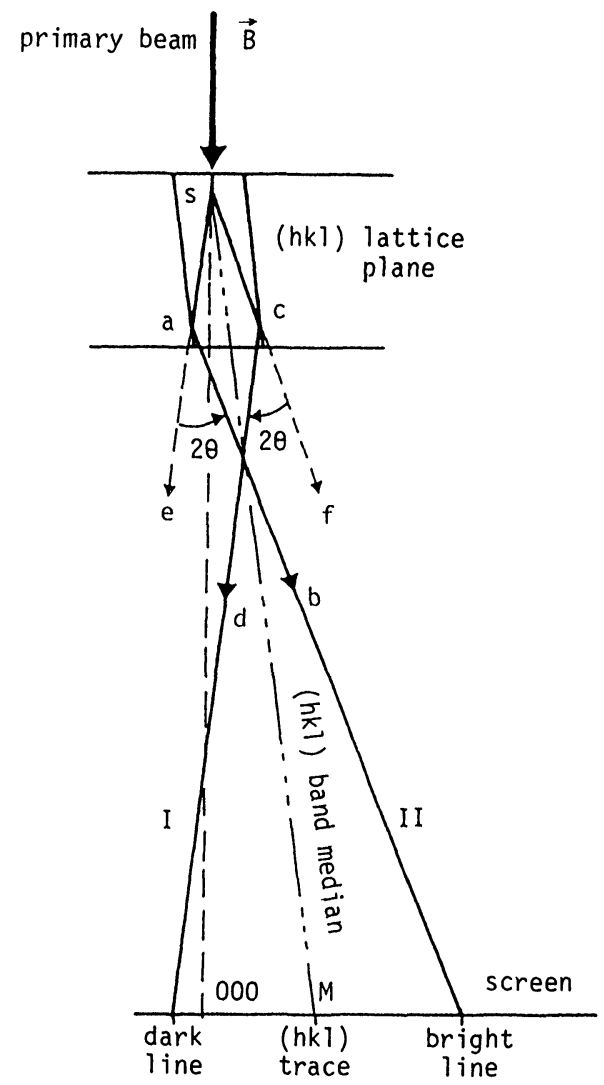

Figure 1 Schematic illustration of the formation of a dark and a bright Kikuchi line. 
1984) is required while the geometry of Kikuchi lines can be well interpreted by a strongly simplified model (Kikuchi 1928) (Figure 1): They occur as a result of diffuse scattering of the electron beams (incident and diffracted) as they penetrate the sample. The loss of energy by a single scattering event is very small and hence the increase in wavelength is negligible. Even if a selected lattice plane does not fulfill the Bragg condition for the primary beam, there are diffuse electrons which can be diffracted from either side of the lattice plane. Those electrons scattered incoherently at sample point $s$ into the direction $\overrightarrow{s a}$ may be Bragg-diffracted by the $h k l$ planes into the direction $\overrightarrow{a b}$, and those from direction $\overrightarrow{s c}$ into direction $\overrightarrow{c d}$. Since $\overrightarrow{s a}$ makes a smaller angle with the incident beam direction than does $\overrightarrow{s c}$, more electrons will be scattered into $\overrightarrow{s a}$ and neighbouring directions than into direction $\overrightarrow{s c}$, and consequently more electrons will be Bragg-diffracted from the direction $\overrightarrow{s a}$ than being diffracted into direction $\overrightarrow{s a}$ through $\overrightarrow{c d}$. The same holds true for all directions lying on a Kossel cone of semi-vertex angle $\left(90^{\circ}-\theta\right)$ with the plane normal as axis of the cone. Hence the Kossel cone II in Figure 2 has an enhanced intensity to form a bright excess Kikuchi line on the fluorescent screen. The lower intensity cone I gives rise to a dark defect line. The intersection of the Kossel cones with the fluorescent screen form hyperbolas. They can be approximated fairly well by straight lines, due to the small values of the Bragg angles, $\theta$, in electron diffraction. It is worth mentioning that the trace of the diffracting planes lies, as a straight line, almost midway between the Kikuchi line pair. Kikuchi patterns are analogous with Kossel patterns in X-ray diffraction.

It can be readily seen from this model that two conditions must be satisfied for Kikuchi lines to be generated. There must be a sufficient number of electrons filling a large angular range along the primary beam direction in order to have a chance to be Braggdiffracted from obliquely oriented lattice planes. Secondly, there must not be significant differences in crystal orientation over the whole diffracting sample volume, otherwise the bright and dark Kikuchi lines would fade away on the background of inelastically scattered electrons. The first condition is met either by extensive scattering of the primary beam when impinging on a thick specimen or, with a thin sample, by a convergent illumination (CBED) which may supply all necessary directions of primary electrons. The 


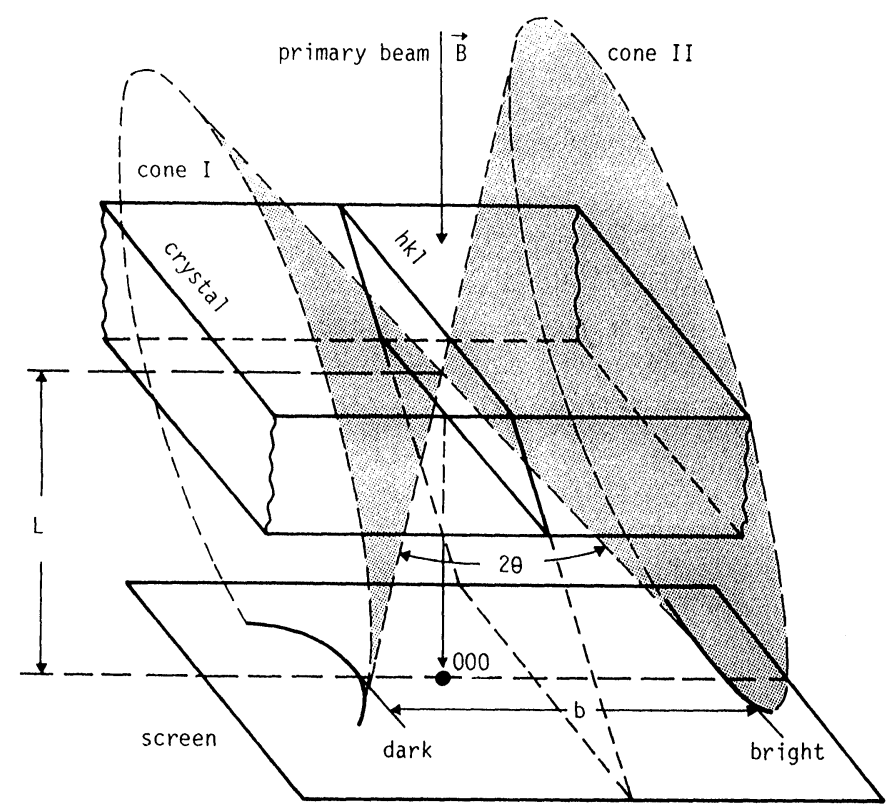

Figure 2 Kossel cones and Kikuchi lines (schematic).

second condition may be fulfilled either with conventional selected area diffraction from large, perfect crystals or, with MBD, with a focussed electron beam of a small spot size, such that the diffracting crystal volume is virtually perfect. When studying samples from heavily deformed materials, it is often essential to decrease the beam spot to less than $100 \mathrm{~nm}$ in diameter in order to find a column of the crystal that is not distorted, and free from dislocations. It is obvious that, by scattering the incoming electron beam, the foil thickness imposes a lower limit on the useful beam size and hence on the tolerable strains. In practice an accuracy of orientation better than $0.5^{\circ}$ may be obtained from Kikuchi patterns related to grains in the same sample even with deformed materials. When evaluating the crystal orientation with respect to the workpiece, a larger error than this is introduced by inaccurate marking of the reference directions when taking the sample from a bulk material, by deviations of the alignment on the sample holder or by local bending of the sampled foil in the TEM. 


\subsection{The determination of grain orientations with the scanning electron microscope (SEM)}

With an SEM grain orientations can be determined from electron backscattering and channelling patterns. As with Kikuchi patterns, sharp channelling or backscattering patterns are only formed if the diffracting volume is a perfect crystal. The blurr of the patterns is a direct measure of the local strain. A bibliography on the observation of crystalline materials by use of diffraction effects in the SEM is given by Joy and Newbury (1977).

2.3.1. For the acquisition of electron backscattering patterns (Figure 3) in the SEM (Venables and Harland 1973) either a photographic plate or a fluorescent transmission screen is placed right in front of the tilted sample. In the latter arrangement the pattern may be recorded by photography or a TV camera through a window from outside. The stationary primary electron beam is incident on the sample at an angle of typically $30^{\circ}$. The backscattered electrons, forming the pattern, have sufficient energy to directly excite the photographic emulsion or fluorescent screen. Lateral resolution is close to the primary beam size which is elongated by projection on the inclined surface and widened to

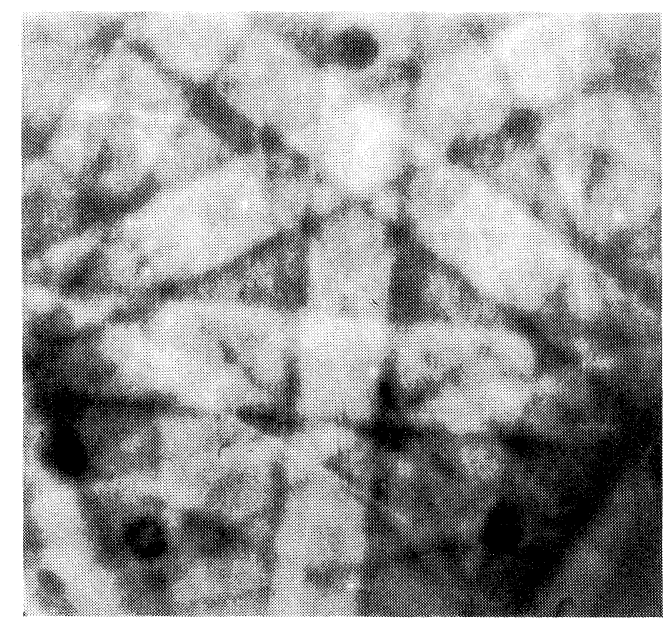

Figure 3 Electron backscattering pattern from copper at $20 \mathrm{kV}$ accelerating voltage. 
some extent by scattering in the sample. Patterns from areas less than $0.5 \mu \mathrm{m}$ diameter have been reported. Advantages of electron backscattering patterns are the very large range of recorded angles larger than $20^{\circ}$ (which facilitates the consistent evaluation of the orientation merely from zone-axis positions), the improved sharpness of lines due to the superior resolution of photographic emulsions or fluorescent screens over electronic scanning systems, less distorted patterns without image rotation due to a lensless geometric projection of the pattern and high probe brightness due to the large aperture of the primary beam. The focus settings and aperture stops of the microscope need not be altered when changing from imaging to backscattering electron diffraction mode. The beam spot has only to remain fixed on the selected grain which is similar to spot analysis in energy dispersive X-ray microanalysis (EDX). Crystal orientations of contiguous grains can be obtained with an accuracy of about $0.5^{\circ}$, a value which approaches the accuracy of Kikuchi patterns. Crystal orientations with respect to a frame fixed to the workpiece can be determined to about $1-2^{\circ}$, since the positions of the samples on the stage and the primary electron beam may vary in this order of magnitude.

2.3.2. Selected area channelling patterns (Coates, 1967; Brooker, 1970) from bulk samples can be generated using either a rockingbeam or a rocking-specimen technique. The patterns are produced by the variation of the backscattered intensity with the angle between the incident beam and the crystal lattice. In the rocking beam method with pre-lens deflection, the scanning facility is used in combination with the probe-forming lens to scan the angle of the incident beam, with the pivot point resting on the sample surface. For larger rocking angles the beam passes through the lens far from the optical axis. Mainly because of the spherical aberration and defocussing errors, the pivot point is widened to a disk. Due to the long focal lengths and large spherical aberration coefficients of the probe-forming lenses and the need for rocking angles in excess of $5^{\circ}$, the illuminated sample area is hardly smaller than $10 \mu \mathrm{m}$ in commercial scanning microscopes. This is not sufficient for the study of deformed materials. Spot sizes in the range of $1 \mu \mathrm{m}$ diameter (this corresponds to the minimum region selected in SAD, see 2.1) are possible by a modification (Joy and Newbury, 1972). The beam 


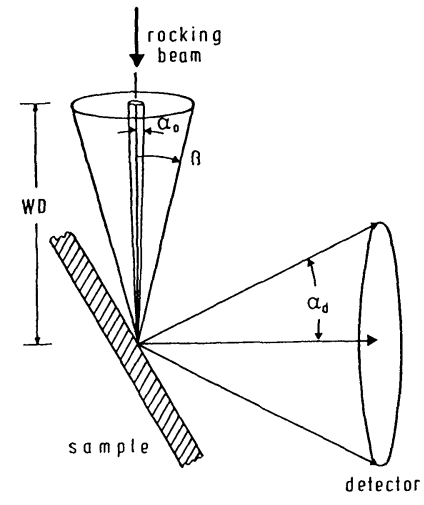

Electron Channelling Pattern

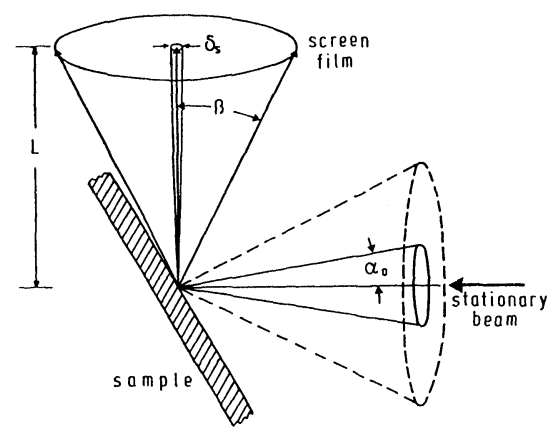

Electron Backscattering Pattern

Figure 4 The correspondence of electron channelling (left) with backscattering (right) patterns.

is scanned on a helix and the probe-forming lens is refocussed according to the inclination between the beam and the optical axis such that the pivot point remains stationary. With the rockingspecimen setup the sample is tilted mechanically under a stationary beam, using a eucentric goniometer stage. A large angular range of about $20^{\circ}$ at a lateral resolution of $10 \mu \mathrm{m}$ is reported by Brunner and Niedrig (1979).

Due to the distortions of the scanning and recording systems, the accuracy of measured orientations using this technique is reduced to values close to those of backscattering patterns. It is obvious from Figure 4 that channelling and electron back-scattering patterns are equivalent. The camera length, $\mathrm{L}$, the beam aperture, $\alpha_{0}$, the acceptance angle, $\beta$, and the resolution, $\delta_{s}$, of the film or fluorescent screen to record the electron backscattering pattern correspond with the working distance, WD, the acceptance angle, $\alpha_{d}$, of the detector, the beam aperture, $\alpha_{0}$, and the maximum scan angle, $\beta$, for the channelling pattern, respectively.

\section{EVALUATION OF ELECTRON DIFFRACTION PATTERNS}

The pairs of Kikuchi or channelling lines are fixed to their crystal lattice planes so that the whole system of lines will exactly follow 
any rotation or tilt movement of the crystal. Precise measurement of orientations relies on this feature and the sharpness of lines. In contrast, electron diffraction spots - which have been widely used for orientation determinations - virtually maintain their positions in the diffraction pattern over a large tilting range of the sample while only changing their intensities. The precise grain orientation can be obtained by simply measuring the position of at least three Kikuchi or channelling bands in a diagram, whereas with spot patterns allowance must be made for the positions and intensities of several spots. Usually lattice planes from different crystallographic zones contribute to a Kikuchi, channelling or backscattering pattern. Hence, unlike spot patterns, they generally are not symmetric with respect to the centre. The orientation of the crystals with respect to a reference frame (e.g. an orthogonal frame with the $x$ - and $y$-axes normal to the beam direction) can be unambiguously determined with high precision by evaluating only one diagram. In general, however, two complementing spot patterns, which must be taken at known specimen tilts, are required. A further advantage of Kikuchi, channelling and backscattering patterns over spot patterns is the wide range of useful sample thicknesses. Even with thick foils close to the limit of transparency, Kikuchi patterns show sufficient contrast. For the study of thin foils, it is advisable to lower the beam voltage and to increase the beam convergence as far as possible, since the formation of Kikuchi patterns is favoured by both means. Furthermore, surfaces of bulk samples can be studied with the SEM.

The wide application of Kikuchi, channelling and backscattering patterns, particularly those of non-cubic symmetry, is impeded by the troublesome procedure of indexing. Only when the primary beam is incident close to a low-index direction do the patterns become so simple that they may be identified by visual inspection. Several procedures have been reported for the evaluation of Kikuchi (Newbury and Joy, 1971; Heilmann et al., 1982; Hartig, 1984) and channelling (Ishida et al., 1981) micrographs. The measurement of diffraction coordinates has been facilitated by using a digitizing tablet, a projection device or a TV camera with image-analysing facility. The orientation determinations have been carried out off-line from the microscope with a separate computer. The on-line data acquisition and evaluation of diffraction spot (Carr 
1982) and Kikuchi or channelling patterns (Schwarzer and Weiland, 1984a; Weiland and Schwarzer, 1986) with a microcomputer linked on-line to the microscope is a significant improvement, since laboratory computers are frequently available (e.g. from the EDX facility).

An electron backscattering (Figure 3), channelling (Figure 5a) or Kikuchi (Figure 5b) pattern contains all the necessary information on the diffracting crystal. The band width, i.e. the distance between two parallel lines corresponds to twice the Bragg angle, $\theta$, and the median represents the lattice plane trace. The angle of tilt between a set of lattice planes and the primary beam direction can be deduced from the distance between their median and the primary beam spot. The geometrical appearance of these diffraction patterns is so similar that virtually the same evaluation procedures can be applied. The program ORKID (ORientations from KIkuchi Diagrams) (Schwarzer and Weiland, 1984b) requires the following input data: the Bravais lattice and-only required for non-cubic crystals-the lattice constants, e.g. c/a ratio for tetragonal and hexagonal crystals. The diffraction-pattern coordinates, i.e. the primary beam position and two points on either line of at least three bands, may be measured on-line. Program execution becomes faster if the lattice constants, the orders of diffraction, and rough estimates, as indicated on most microscope panels, of the beam voltage and the camera length are supplied. Sets of $h k l$ lattice planes are selected from those of which interplanar spacings correspond, within some error limits, with the band widths according to Bragg's law and from interplanar angles that are consistent with angles measured between the bands. By using formulas of fundamental crystal geometry and the positions of at least three bands, the orientation of any selected reference direction (e.g. rolling, normal and transverse direction) can be expressed in terms of Miller indices with respect to the frame of the diffracting crystal. In rare cases more than one solution is found. This may be due to small errors in measurement. Even without such errors, however, several solutions may be consistent with the few bands supplied to the computer, but not with the complete Kikuchi pattern. In order to distinguish the correct solution, the Kikuchi maps for the possible solutions are calculated and displayed, along with the measured data, on the monitor screen (Figure 5c). The graphical display of 

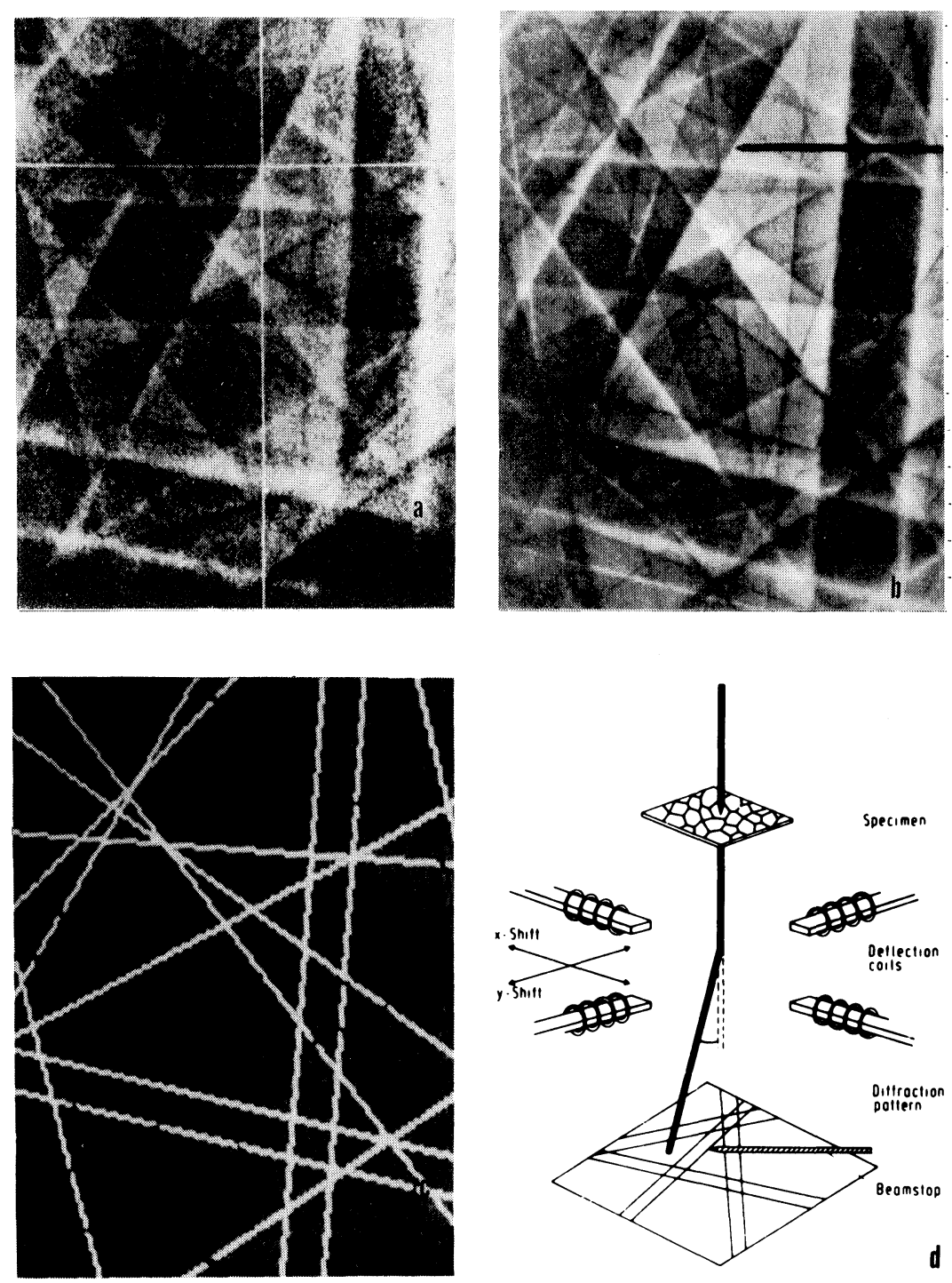

Figure 5 The on-line evaluation of Kikuchi and channelling patterns: (a) channelling pattern (b) Kikuchi pattern from a ferrite grain at $150 \mathrm{kV}$ (c) recomputed pattern for the ferrite grain of (a) and (b) (unit vector in beam direction $\langle 0.250 .470 .85\rangle$ ) (d) schematics of on-line acquisition of diffraction patterns with the TEM. 
the solution serves as a check of reliability. The computation is typically executed in less than 1 minute for cubic indices up to $\{800\}$. The output data of the ORKID program are: The Laue indices of the measured bands, the normalized Miller indices of an orthogonal reference frame whose $z$-axis coincides with the reverse primary beam direction, and the Euler angles. This program has been used equally well for the indexing of channelling and electron backscattering patterns, in addition to Kikuchi patterns.

\section{ON-LINE DATA ACQUISITION}

Diffraction data may be acquired on-line with the TEM by simply deflecting the pattern over the fluorescent screen until selected features (diffraction spots or Kikuchi lines) coincide with a reference mark (Figure 5b). The voltage drop over the beam deflection coils is passed directly on to the computer, as a measure of the selected position coordinates in the pattern. With the SEM a similar procedure is possible by placing a cursor at selected positions on channelling or electron backscattering patterns displayed on the monitor (Figure 5a). The operator using this semi-automated method has to select the coordinates with some skill, which is comparable with the skill required when selecting characteristic X-ray lines in quantitative EDX analysis. This is not really a disadvantage since experience speeds up analysis and can only be replaced to some extent by more sophisticated hard- or software. Furthermore a fully automatic on-line evaluation is susceptible to erroneous data and is limited by the memory size of present laboratory computers. Nevertheless some efforts have been made to automatically transmit the pattern from the microscope to the computer (Ishida et al., 1984; Neumann, 1987).

In quantitative texture analysis the distribution of grain orientations is considered by number as well as by volume. The volume fraction and orientation of each grain is required. It is a unique advantage of electron microscopy that complementary studies of diffraction and imaging techniques are possible on the same specimen area. Hence the surface and, making reasonable assumptions, the volume fraction of grains with a specific orientation can be determined simply by a stereological evaluation of the TEM or SEM image. To this end the grain-boundary coordinates may be 


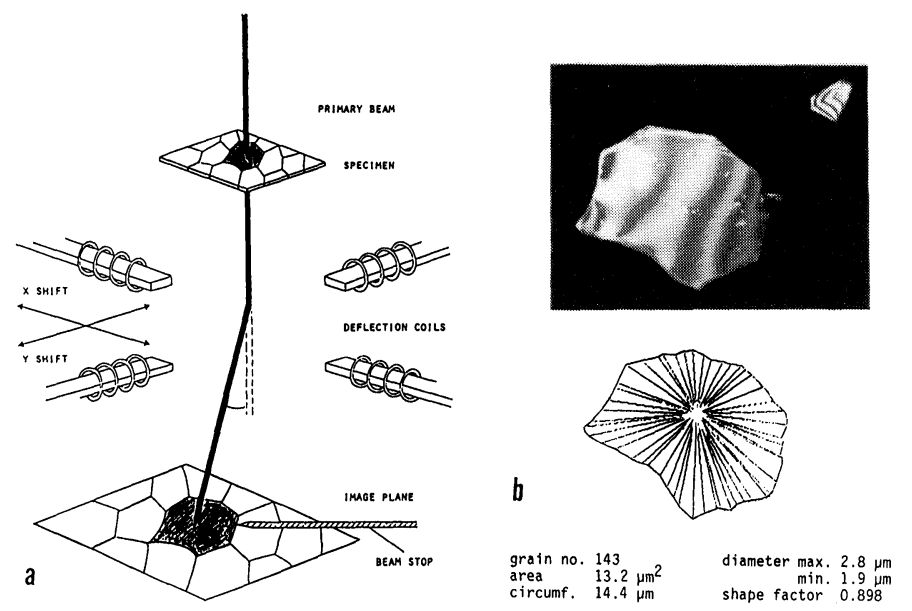

Figure 6 (a) On-line acquisition of stereological data (b) Display of the measured grain boundary indicating the triangular sections for grain-size calculation.

acquired on-line, in a similar way to which the data input is obtained from diffraction patterns, by deflecting the TEM image with respect to a mark on the screen (Figure 6) (Weiland and Schwarzer 1985).

\section{ODF AND MODF REPRESENTATION OF INDIVIDUAL GRAIN ORIENTATIONS}

For comparison of individual grain orientation with X-ray polefigure measurements it is convenient to compute the series expansion of the orientation distribution function (ODF) (Bunge, 1982)

$$
f(g)=\sum_{l=0}^{L} \sum_{\mu=l}^{M(l)} \sum_{v=l}^{N(l)} C_{l}^{\mu v} \dot{T}_{l}^{\mu v}(g)
$$

The truncation error, which arises when using Dirac functions for the series expansion of a discrete set of individual orientations, can be avoided by replacing each Dirac function by an analytic (Gauss) function centered at each particular orientation $g$ (Wagner, 1986). The expansion coefficients are then expressed by

$$
C_{l}^{\mu v}=\frac{\exp \left(-l^{2} \phi_{0}^{2} / 4\right)-\exp \left(-(l+1)^{2} \phi_{0}^{2} / 4\right)}{1-\exp \left(-\phi_{0}^{2} / 4\right)} \cdot \sum_{i=1}^{N} V_{i} \cdot \dot{T}_{l}^{\mu v}\left(g_{i}\right)
$$


where $V_{i}$ stands for the volume fraction of grain $i, 2 \cdot \phi_{0}$ for the full width at $1 / e$ maximum of the Gaussian peak. The optimum $\phi_{0}$ value depends-not very sensitively-on the number of measurements $N$ and the sharpness of the texture. For most applications $\phi_{0}$ values between $5^{\circ}$ and $10^{\circ}$ are appropriate.

Similarly the orientation correlation functions between contiguous grains of the same or of different phases can be calculated (Bunge and Weiland, 1987). The distribution of special grain boundaries is revealed which may significantly affect the material properties. Orientation differences $\Delta g$ are specified in Euler angles. For the series expansion each orientation difference is superimposed by a Gaussian shaped peak. The physical misorientation function is:

$$
F^{\alpha \beta}(\Delta g)=\sum_{l=0}^{L} \sum_{\mu=l}^{M^{\alpha}(l)} \sum_{v=l}^{M^{\beta}(l)} D_{l}^{\mu \nu} T_{l}^{\mu \nu}(\Delta g)
$$

The correlation function $\varphi^{\alpha \beta}(\Delta g)$ is then obtained by normalizing the physical misorientation function $F^{\alpha \beta}(\Delta g)$ with respect to the uncorrelated misorientation function

$$
\varphi^{\alpha \beta}(\Delta g)=\frac{F^{\alpha \beta}(\Delta g)}{F_{\mu}^{\alpha \beta}(\Delta g)}
$$

The uncorrelated misorientation function can be computed either from "random pairs" or as the convolution function of both textures:

$$
F_{\mu}^{\alpha \beta}(\Delta g)=\int_{V} f^{\alpha}(\Delta g \cdot g) \cdot f^{\beta}(g) d g
$$

\section{APPLICATION ON DUAL-PHASE STEEL}

A dual-phase steel of $20 \%$ by volume martensite was studied. The structure was obtained by cold rolling and intercritical open-coil annealing of low-carbon steel $(0.05 \% \mathrm{C}, 0.44 \% \mathrm{Si}, 2.55 \% \mathrm{Mn})$ (Vlad, 1982). Specimens in this initial state, and after a tensile elongation for $14 \%$ were prepared for the TEM. The orientation and size of approximately 100 ferrite and 200 martensite grains were determined for material in the two conditions.

The study of individual grain orientations in dual-phase steel with 
the TEM is particularly difficult since

1) the sample is magnetic, which gives rise to misalignments and forbids large specimen tilts,

2) the tetragonal martensite lattice differs only slightly from the bcc ferrite lattice, and

3) martensite has a high degree of internal strains which affects the sharpness of Kikuchi lines.

The high local resolution is an outstanding advantage of TEM orientation measurements and allows investigations not possible with any other technique. Ferrite and martensite lattices differ only slightly especially at this low carbon content and thus their diffracted reflections cannot be resolved by conventional diffraction techniques. X-ray and neutron pole-figure measurements only yield the mean texture of both phases, whereas with the TEM each grain can be classified as either ferrite or martensite. The ferrite thins more rapidly during electrolytic polishing than the martensite. Martensite remains thick and hence appears darker in the TEM image as compared to the ferrite grains. Furthermore the Kikuchi pattern of the martensite is diffuse due to internal strains while ferrite grains show sharp patterns. Grain after grain can be analysed by focussing the primary beam spot on the selected area to obtain its Kikuchi pattern.

While the determination of orientation in the ferrite presents no problems, the study of martensite is restricted by two operating parameters of the TEM. For the generation of Kikuchi patterns it is necessary that the electron beam be fine enough in order to sample a column of sufficiently perfect crystal. This requires a beam of approximately ten nanometer in diameter, and the sampled area must be so thin that the beam will not be widened unduly by scattering. As the area of crystal becomes less perfect, the resulting Kikuchi pattern is increasingly blurred until at some stage it is no longer possible to distinguish the individual lines. Furthermore, the contribution of neighbouring martensite platelets to the pattern must be avoided. At the same time a minimum intensity in the pattern, which can be achieved only by increasing the beam diameter, is necessary in order to be able to evaluate it. The two parameters are mutually exclusive and a compromise must be 
reached. The advantage of the on-line procedure becomes apparent in this situation. Since the fluorescent screen can reproduce much larger intensity differences than photographic material, even diffuse Kikuchi lines can be evaluated and, making allowance for larger limits of experimental error and checking the possible solutions with the recomputed maps, correct orientations are found.

Figure 7 shows a TEM micrograph of intercritically annealed dual-phase steel which has been inserted in a schematic microstructure plot composed of the ferrite-grain Kikuchi maps. The sample was taken from the longitudinal plane. This example illustrates that a reasonably large number of grains can be studied even with high-density materials provided that adequate preparation techniques and high accelerating voltages are used. The martensite is found to form chains of small "islands" located at the grain boundaries of the ferrite. It has an inhomogeneous striped appearance which corresponds to lath martensite (Krauss and Marder, 1971). Within each martensite island, up to 10 different orientations can be found, depending on the size of the grain and number of its neighbours. Figure 8 shows the ODF for 100 ferrite grains and Figure 9 the ODF for 217 martensite grains obtained by individual grain orientation measurements. Thereby the volume fraction of the grains have been taken into account according to Eq. (4). For the

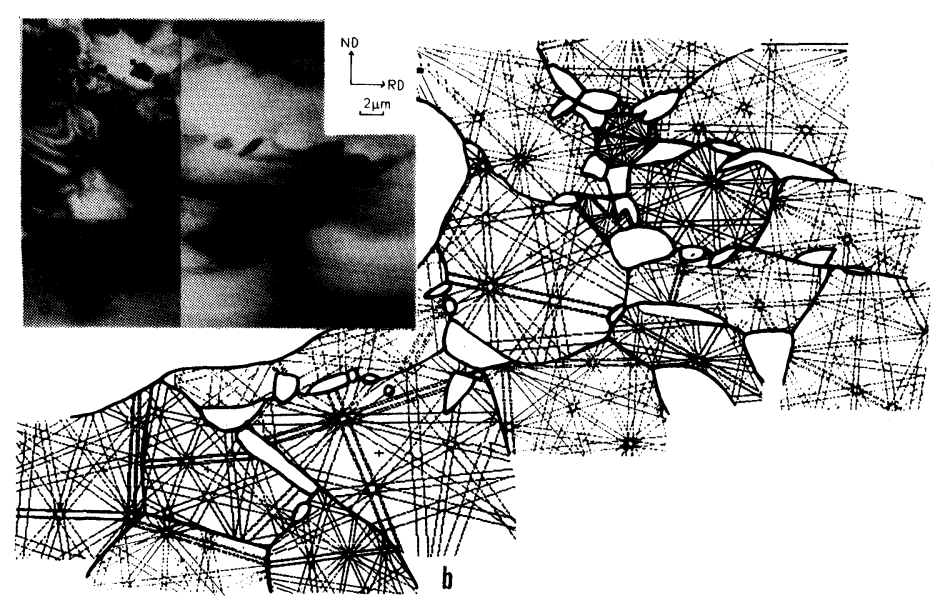

Figure 7 Intercritically annealed dual-phase steel. (a) TEM micrograph (b) Schematic microstructure composed of ferrite grain Kikuchi maps. 

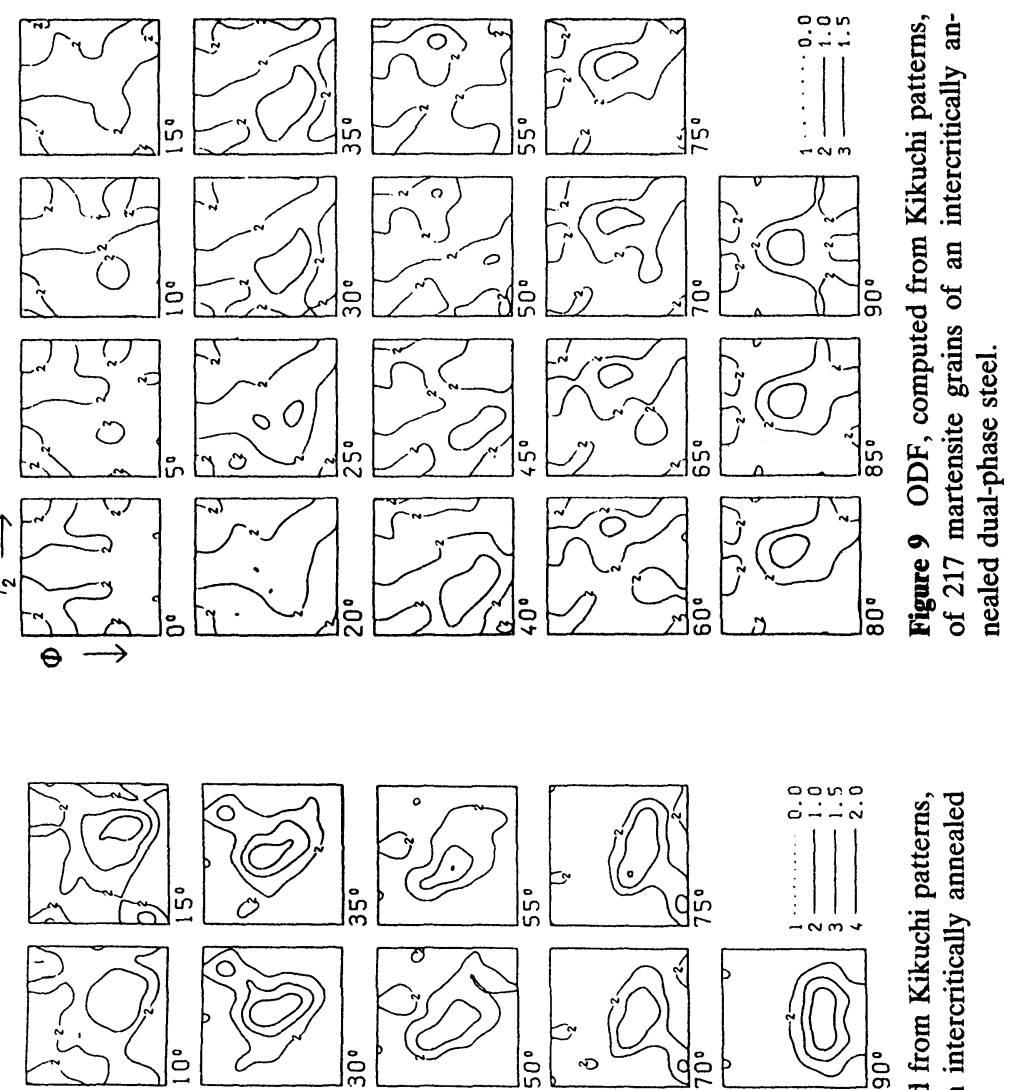

憘
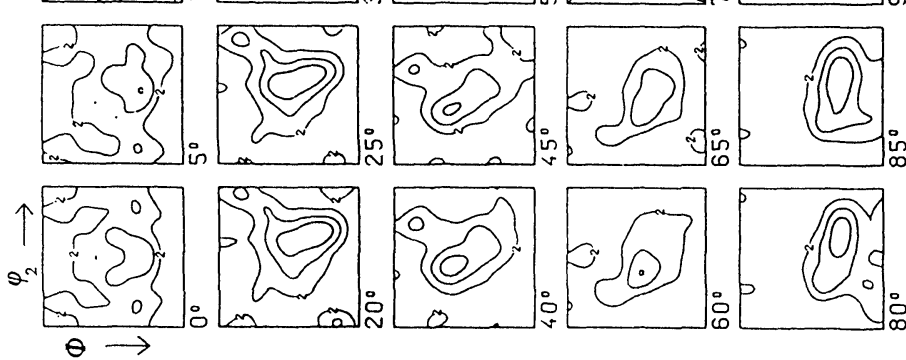

프

옹 엉

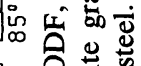

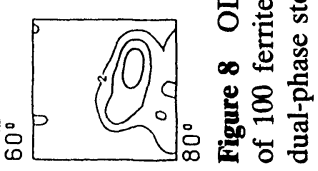


calculations of ODF the Euler angles have been expressed, as usual, in terms of the Miller indices $(h k l)$ for the rolling plane and [uvw] for the rolling direction, using the equations $2.57,2.58$ and 2.59 in (Bunge 1982).

After the intercritical annealing (initial state), the texture of the ferrite phase (Figure 8) is similar to the typical recrystallisation texture of low carbon steels. The main component is a fibre deviating $11^{\circ}$ from the $\langle 111\rangle$-fibre. Here the $\langle 110\rangle$-fibre, typical for iron recrystallisation textures, is not well developed. An additional $(211)\langle 131\rangle$ component appears in the ODF, with the same intensity as the $\langle 111\rangle$-fibre. The martensite shows only a weak texture (Figure 9). Due to the 24 possible austenite- martensite orientation relationships, it is, over a wide range, nearly random. Two main components exist in this ODF, one is rotated $5^{\circ}$ from $(112)\langle 111\rangle$, and the other one lies close to $(232)\langle 111\rangle$.

The tensile elongation of $14 \%$ changes the textures of both phases. In the ferrite texture (Figure 10) the main component is shifted towards the $\langle 111\rangle$-fibre axis and is now $7^{\circ}$ away from it. The greatest change in texture after deformation is observed in the martensite. Compared to the nearly random orientation distribution in the initial state, a well developed texture now appears (Figure 11) similar to the typical iron deformation texture. Like the ferrite, the martensite texture consists of a fibre close to $\langle 111\rangle$ and a strong (211) $\langle 131\rangle$ component.

The reason for this phenomenon may be seen in the morphology of the martensite itself. The microstructure of a martensite island consists of groups of packets. Each packet, made up of a large number of laths and showing a high dislocation density, has its individual orientation. Typical diameters for the packets are 0.2 to $1 \mu \mathrm{m}$. Orientation-correlation studies between martensite packets, after the intercritical annealing, reveal peaks from low-angle boundaries and a weak component due to a $\Sigma 3$ coincidence relationship (Figure 12). A further component in the MODF is the Bain relationship which results from the austenite-martensite transformation. Auger microanalysis in the ferrite and martensite phase revealed no significant differences in the carbon content $(0.05 \mathrm{wt} . \%)$. This low-carbon martensite is not as hard as ordinary plate martensite. The yield strength is only three times higher than that of pure ferrite. According to the observed texture develop- 

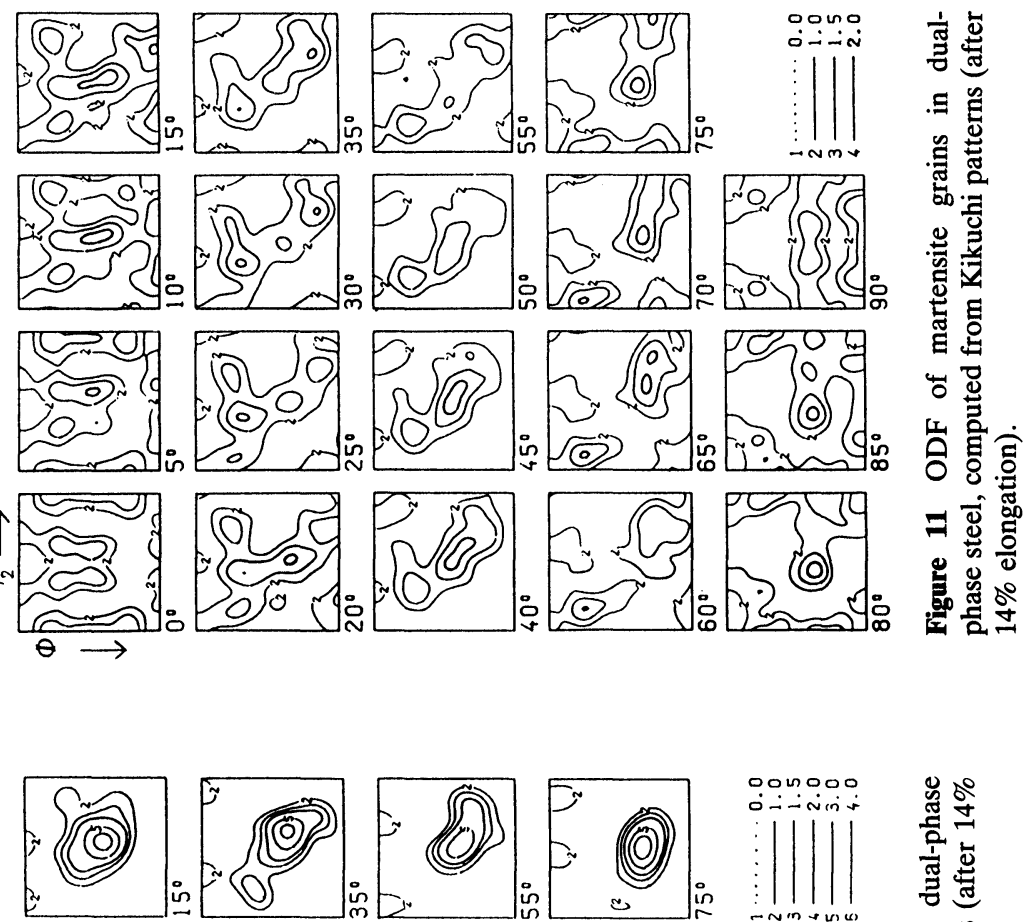

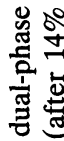
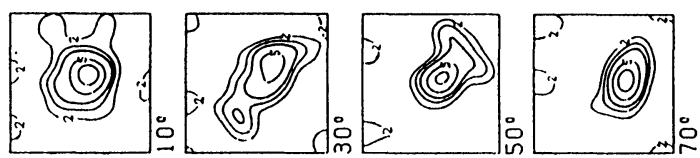

$\because$ (1)

.
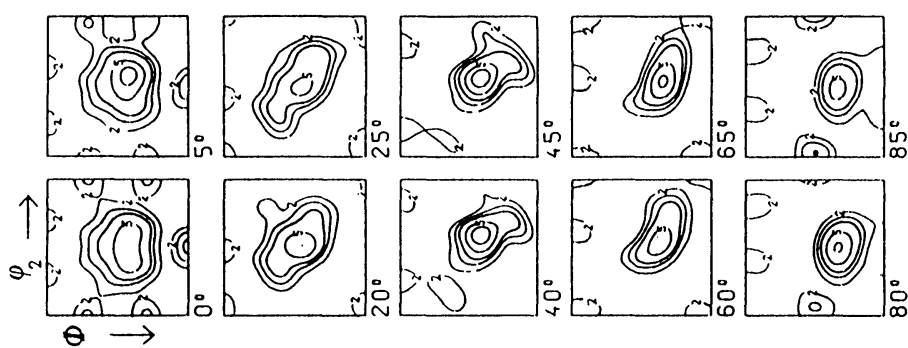

을 흥

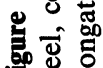




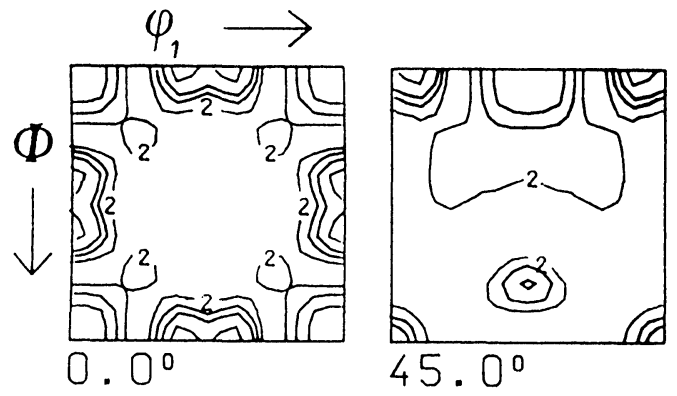

Figure 12 MODF between contiguous martensite-martensite packets after intercritical annealing.

ment, the conclusion is drawn that the martensite in this material is deformed along with the ferrite, but at a lower deformation rate. The orientation relationship between both phases in the initial state (Figure 13) shows low-angle boundaries and the Bain relationship. This is due to the formation of the austenite from the ferrite and the subsequent austenite-martensite transformation. After the tensile elongation, the Bain relationship has disappeared (Figure 14), but a $\Sigma 3$ component along with another component, which is due to a rotation of $62.8^{\circ}$ around $\langle 110\rangle$, occurs.

As an illustration of the insufficient accuracy of SAD orientation measurements, the ferrite and martensite grain orientations from Kikuchi measurements have been converted into hypothetic SAD
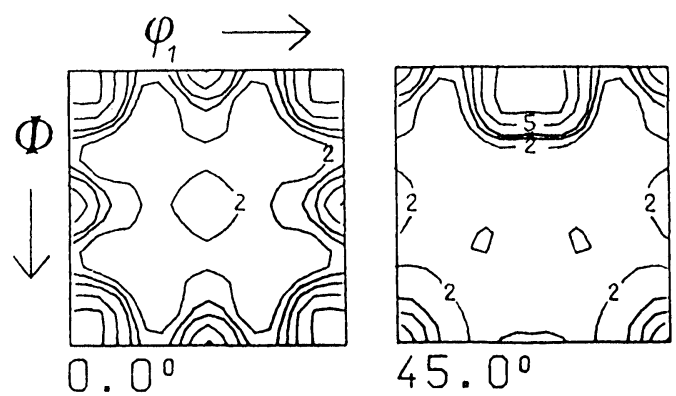

Figure 13 MODF between contiguous ferrite and martensite grains after intercritical annealing. 

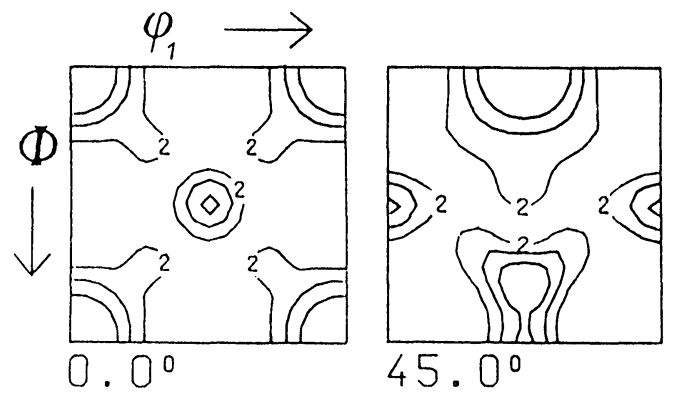

Figure 14 MODF between contiguous ferrite and martensite grains after $14 \%$ elongation.

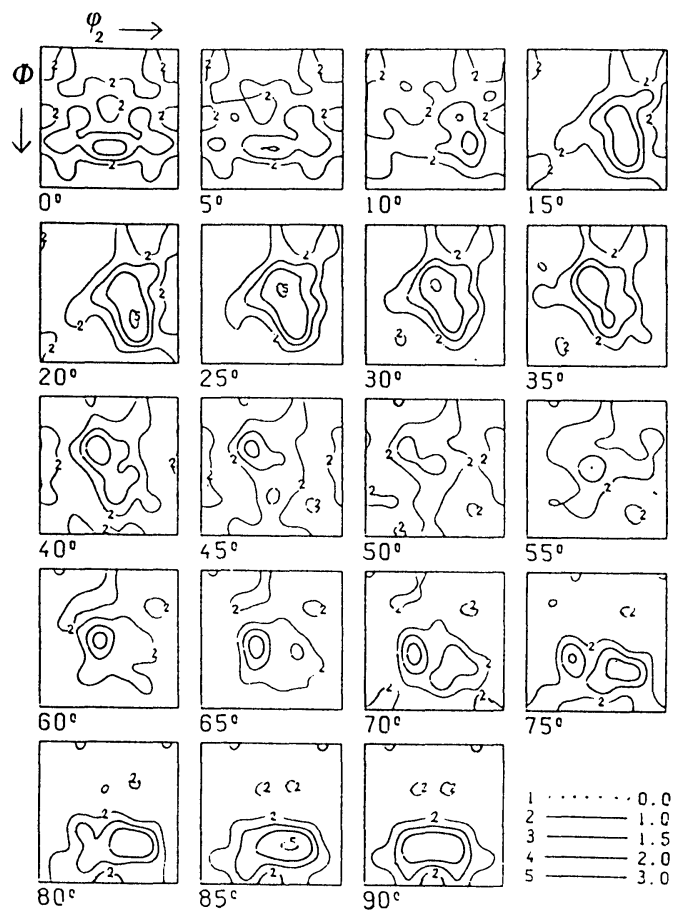

Figure 15 ODF calculated from simulated (SAD) orientations of ferrite grains of a dual-phase steel after intercritical annealing. 
orientations by rounding off each exact primary beam direction to the nearest integer direction (under the assumption that spots up to $\{410\}$ were available for the interpretation of spot patterns). In spite of the fact that the SAD orientations differed less than $9^{\circ}$ from the original Kikuchi orientations, the ODF is significantly changed (cf. Figures 8 and 9 with Figures 15 and 16). When evaluating the ODF from SAD spot patterns these falsifications may not always be noticed. Particularly with the weak martensite texture, an ODF similar to the well-known (and often expected) recrystallisation texture is obtained, which differs significantly from the correctly measured texture. The conclusion reached is that SAD orientation measurements should be replaced by the Kikuchi method particularly if orientation distributions and orientation correlations are studied.

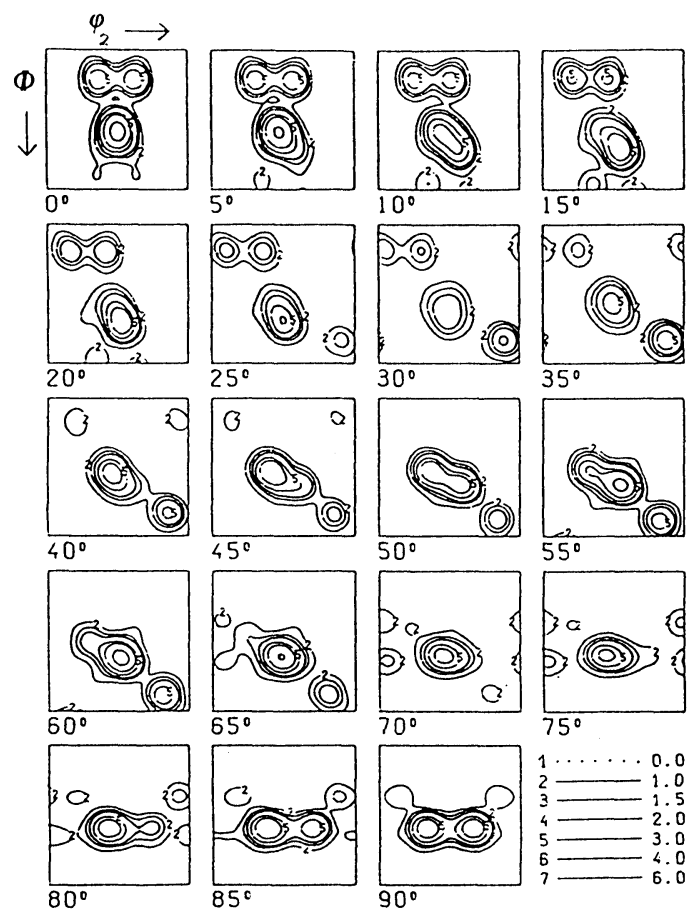

Figure 16 ODF calculated from simulated (SAD) orientations of martensite grains of a dual-phase steel after intercritical annealing. 

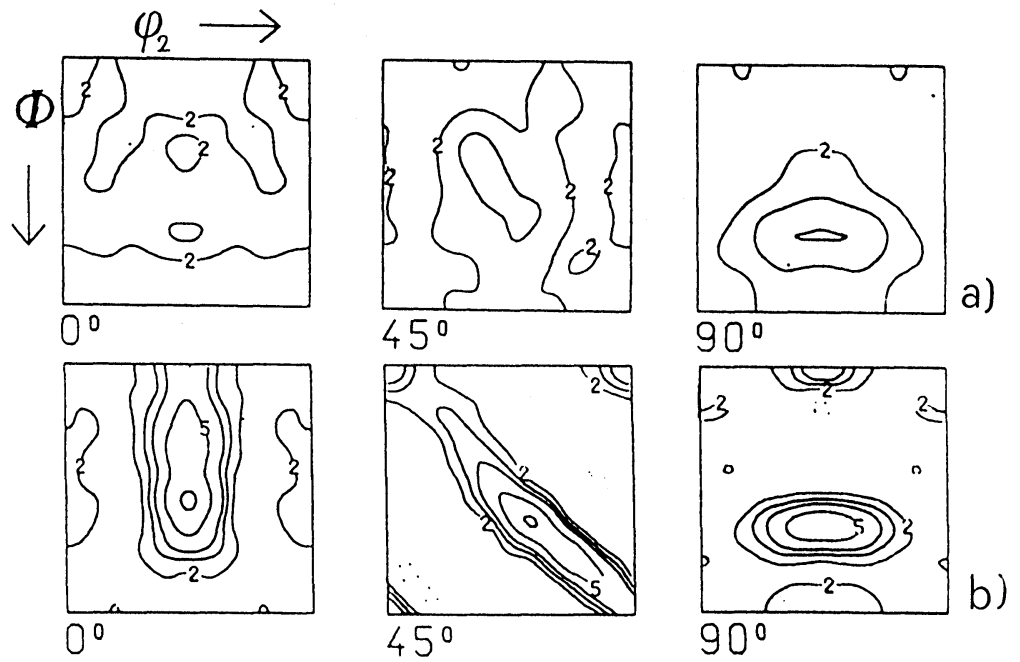

Figure 17 ODF of the dual-phase steel after intercritical annealing, computed from (a) electron diffraction (b) neutron diffraction pole-figure measurements.
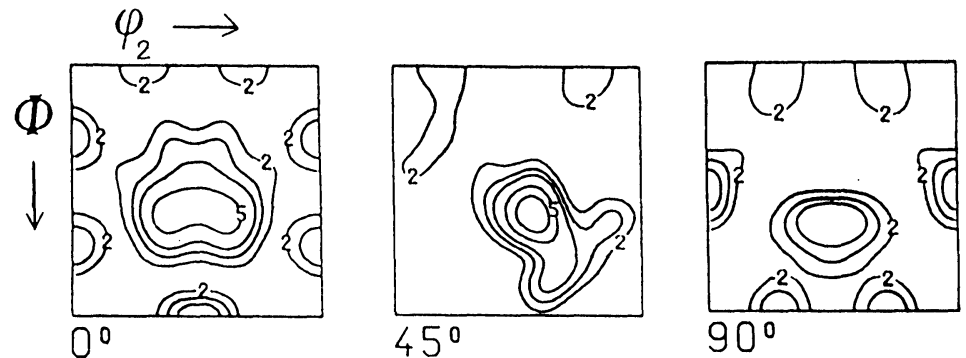

a)
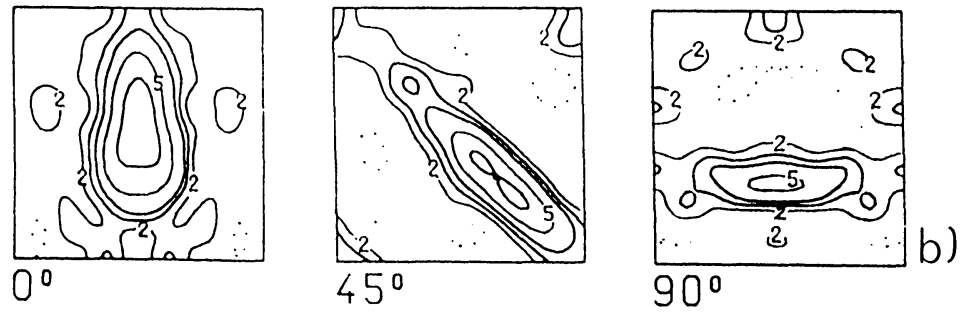

Figure 18 ODF of a dual-phase steel after 14\% elongation, computed from (a) electron diffraction (b) neutron diffraction pole-figure measurements. 


\section{COMPARISON WITH X-RAY AND NEUTRON DIFFRACTION MEASUREMENTS}

Since martensite cannot be distinguished from ferrite by X-ray or neutron diffraction, the average ODF of both phases, according to their volume fraction, is obtained from pole-figure measurements. For a comparison with the electron diffraction measurements, ODF's were composed of the individual orientations of both phases, taking the volume fraction of each phase into account but not distinguishing between the phases. These composed ODF's (Figures $17 \mathrm{a}$ and 18a) correspond well with the average ODF's from pole-figure measurements (Figures 17b, 18b, and 19). The differences may result from the higher statistical significance of

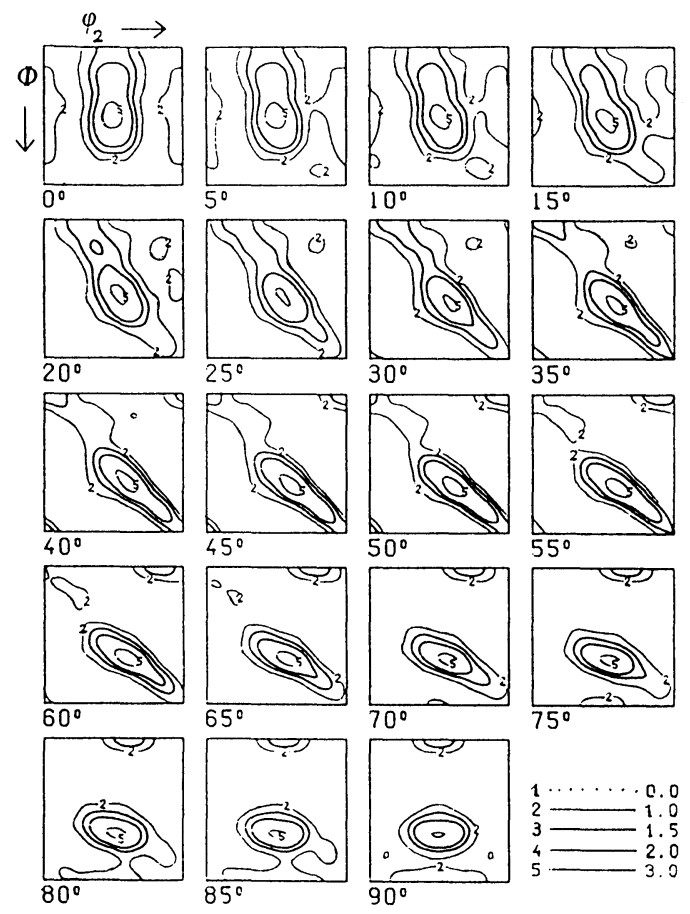

Figure 19 ODF, computed from X-ray pole-figure measurements, of an intercritically annealed dual-phase steel. 
X-ray and particularly neutron diffraction measurements which represent a considerably greater volume fraction than the individual grain orientation measurements by electron diffraction. Electron diffraction yields the local texture of a small volume fraction which may differ from the global texture of the workpiece.

Without a priori information of the separate texture components of ferrite and martensite, the individual textures of both phases cannot be reconstructed from pole-figure measurements, while the average texture can be estimated from its components as obtained by individual grain orientation measurements. In the initial state the martensite and the ferrite textures are distinctly different (Figure 8 and Figure 9) whereas the ODF calculated from X-ray and neutron diffraction measurements (Figures $17 \mathrm{~b}$, and 19) is similar to the ferrite texture. This results from the relatively strong ferrite texture dominating the comparatively weak martensite texture, and from the higher volume fraction of the ferrite. However, after deformation the textures of ferrite and martensite (Figures 10 and 11) are very similar and therefore, the average ODF as obtained from pole-figures (Figure 18b) cannot differ much from the mean ODF of both components (Figure 18a). From this example it follows that, in general, no conclusions on the individual ferrite and martensite textures of dual-phase steels can be drawn using pole-figure measurement techniques.

\section{Acknowledgment}

The authors gratefully acknowledge the loan of the equipment and financial support from the German Ministry of Research and Technology (BMFT; Project 03S3390). The authors wish to express their appreciation for encouragement and helpful suggestions from H.-J. Bunge. Thanks are due to S. Möller, Institut de Métallurgie Structurale, Université Neuchâtel, for contributing the electron backscattering pattern of Figure 3, and to I. P. Welch, for the neutron diffraction measurements.

\section{References}

Brooker, G. R. (1970) In: Amelinckx, S., Gevers, R., Remant, G., and Van Landuyt, J., (eds.), "Modern Diffraction and Imaging Techniques in Materials Science", North-Holland Publ. Company, Amsterdam-London, pp. 613-653.

Brunner, M. and Niedrig, H. (1979) Beitr. elektr. Direktabb. Oberfl. (BEDO) 12/1, 333-338.

Bunge, H.-J. (1982) Texture analysis in material science. Butterworths London.

Carr, M. J. (1982) JEOL news 20E, 7-9. 
Coates, D. G. (1967) Phil. Mag. 16, 1179-1784.

Hartig, Ch. (1984) Elektronenmikroskopische Untersuchung der DeformationsTexturen und des Beginns der Rekristallisation von plastisch verformten Ni-Einkristallen. Ph. D. Thesis, TU Berlin.

Heilmann, P., Clark, W. A. T., and Rigney, D. A. (1982) Ultramicroscopy 9, 365-372.

Ishida, Y., Mori, M., Arimoto, A. and Onoe, M. (1981) Proc. 6th Intern. Conf. on Textures of Materials, ICOTOM 6, 601-608.

Joy, D. C. and Newbury, D. E. (1981) SEM/1977 Vol. I, Proc. Workshop on Analytical Electron Microscopy, ed. O. Johari (IITRI Chicago), pp. 445-454.

Joy, D. C. and Newbury, D. E. (1972) J. Material Science 7, 714-716.

Kikuchi, S. (1928) Jap. J. Physics 5, 83-96.

Krauss, G. and Marder, A. R. (1971) Met. Trans., 2, 2343-2357.

Laird, C., Eichen, E. and Bitter, W. R. (1966) J. Applied Phys. 37, 2225-2231.

Neumann, P. (1987) MPI Düsseldorf, private communication.

Newbury, D. E., and Joy, D. C. (1971) Proc. 25th Anniversary Meeting of EMAG, Inst. Physics, pp. 306-309.

Riecke, W. D. (1961) Optik 18, 278-293.

Riecke, W. D. (1962) Optik 19, 19-116.

Reimer, L. (1984) Transmission Electron Microscopy, Springer-Verlag BerlinHeidelberg-New York-Tokyo.

Schwarzer, R. and Weiland, H. (1984a) Proc. 7th Intern. Conf. on Textures of Materials, ICOTOM 7, 839-843.

Schwarzer, R. A. and Weiland, H. (1984b) Proc. 8th European Congr. on Electron Microscopy, Budapest, 341-342.

Uyeda, R., Nomoyma, M. and Kogiso, M. (1985) J. Electr. Micr. (Japan) 14, 296.

Venables, J. A. and Harland, C. J. (1973) Phil. Mag. 27, 1193-1200.

Vlad, C. M. (1982) Stahl und Eisen 102, 1101-1106.

Wagner, F. (1986) In: Bunge, H.-J., (ed.), Experimental techniques of texture analysis, DGM-Informations-Ges. Oberursel, pp. 115-123.

Whelan, M. J. (1970) In: Amelinckx, S., Gevers, R., Remant, G., and Van Landuyt, J., (eds), Modern Diffraction and Imaging Techniques in Materials Science, North-Holland Publ. Com., Amsterdam-London, pp. 35-98.

Weiland, H. and Schwarzer, R. (1984) Proc. 7th Intern. Conf. on Textures of Materials, ICOTOM 7, 857-862.

Weiland, H., and Schwarzer, R. (1985) BEDO 18, 55-60.

Weiland, H. and Schwarzer, R. (1986) In: Bunge, H.-J. (ed.), Experimental Techniques of Texture Analysis, DGM-Verlagsges. Oberursel, pp. 301-313. 